\title{
ON $Z_{p}$-EXTENSIONS OF REAL ABELIAN FIELDS
}

\author{
BY AKIRA INATOMI
}

In [1], Fukuda and Komatsu gave a sufficient condition for the $\lambda$ invariants of real quadratic fields to vanish. In this note, we shall show that the results obtained in [1] can be extended in a real abelian case.

Let $k$ be a finite real abelian extension of $Q$ and $p$ an odd prime number which splits completely in $k$. Let $\mathscr{Q}_{1} \mathscr{P}_{2} \cdots \mathscr{P}_{r}$ be the prime factorization of $(p)$ in $k$, where $r=[k: Q]$. A $Z_{p}$-extension

$$
k=k_{0} \subset k_{1} \subset \cdots \subset k_{n} \subset \cdots \subset K
$$

is the cyclotomic $Z_{p}$-extension, since $k$ is a totally real field and Leopoldt's conjecture is valid for $k$. Let $C_{n}$ be the ideal class group of $k_{n}, A_{n}$ the $p$-primary part of $C_{n}, \sigma$ a topological generator of $\operatorname{Gal}(K / k)$ and we denote also by $\sigma$ the restriction of $\sigma$ to $k_{n}$. Let

$$
C_{n}^{\prime}=\left\{c \in C_{n} ; c^{\sigma}=c\right\} \text { and } B_{n}=C_{n}^{\prime} \cap A_{n} .
$$

We have the following result by Greenberg [2], since every $\mathcal{Q}_{\imath}$ is totally ramified in $K$.

LEMMA 1. There exists an integer $n_{0}$ such that $\left|B_{0}\right| \leqq\left|B_{1}\right| \leqq \cdots \leqq\left|B_{n_{0}}\right|=$ $\left|B_{n_{0}+1}\right|=\cdots=\left|B_{n}\right|=\cdots$.

Let $E$ be the unit group of $k$. Then

$$
E=\{1,-1\} \oplus E^{\prime},
$$

where $E^{\prime}$ is a free abelian group of rank $r-1$. We denote by $N_{m, n}$ the norm mapping from $k_{m}$ to $k_{n}(m \geqq n)$. Let $H=N_{n, 0}\left(k_{n}\right) \cap E$, then there exists a base $\left\{\eta_{1}, \eta_{2}, \cdots, \eta_{r-1}\right\}$ of $E^{\prime}$ and positive integers $c_{1}, c_{2}, \cdots, c_{r-1}$ such that

$$
H=\{1,-1\} \oplus\left[\eta_{1}^{c_{1}}, \eta_{2}^{c_{2}}, \cdots, \eta_{r-1}^{c_{r-1}}\right]
$$

and $c_{\imath} \mid c_{\imath+1}$ for $i=1, \cdots, r-2$. Since $\left[k_{n}: k\right]=p^{n}$,

Then we have

$$
H \supset\{1,-1\} \oplus\left[\eta_{1}^{p^{n}}, \eta_{2}^{p^{n}}, \cdots, \eta_{r-1}^{p^{n}}\right] .
$$

Received November 25, 1988. 


$$
c_{1}=p^{n-s_{1}}, c_{2}=p^{n-s_{2}}, \cdots, c_{r-1}=p^{n-s_{r-1}} \text {, }
$$

where $s_{1}, s_{2}, \cdots, s_{r-1}$ are integers such that $n \geqq s_{1} \geqq s_{2} \cdots \geqq s_{r-1} \geqq 0$. Let $l_{n}=$ $s_{1}+s_{2}+\cdots+s_{r-1}$, then $[E: H]=p^{n(r-1)-l_{n}}$.

We denote by $e(\mathscr{P})$ the ramification index of a place $\mathscr{Q}$ of $k$ with respect to $k_{n} / k$. Since $k_{n}$ is a finite cyclic extension of $k$, by Yokoi [3] we have

$$
\begin{aligned}
\left|C_{n}^{\prime}\right| & =\left|C_{0}\right| \times \frac{\Pi_{\mathscr{P}} e(\mathcal{Q})}{\left[k_{n}: k\right][E: H]} \\
& =\frac{\left|C_{0}\right| \times \overbrace{}^{n} \times \cdots \times p^{n}}{p^{n} \times p^{n(r-1)-l_{n}}} \\
& =\left|C_{0}\right| p^{l_{n}} .
\end{aligned}
$$

Hence $\left|B_{n}\right|=p^{c+l_{n}}$, where $c$ is the integer such that $p^{c}$ divides $\left|C_{0}\right|$ exactly. Then, by lemma 1

$$
l_{0} \leqq l_{1} \leqq \cdots \leqq l_{n_{0}}=l_{n_{0}+1}=\cdots=l .
$$

Since $k_{\mathscr{P}_{i}} \cong Q_{p}(1 \leqq i \leqq r)$, if $\varepsilon$ is any element of $E^{\prime}$ which is not equal to 1 , then there exists the positive integer $m_{\varepsilon, \imath}$ for $\mathcal{Q}_{\imath}$ such that $\varepsilon^{p-1} \in 1+p^{m_{\varepsilon, \imath}} Z_{p}$ and $\varepsilon^{p-1} \notin 1+p^{m_{\varepsilon}, i+1} Z_{p}$. Let $m_{\varepsilon}=\operatorname{Min}\left\{m_{\varepsilon, \imath} ; 1 \leqq i \leqq r\right\}$.

LEMMA 2. If $n \geqq m_{\varepsilon}$, then $\varepsilon^{\prime}=\varepsilon^{(p-1) p^{n-m_{\varepsilon}+1}}$ is contained in $H$.

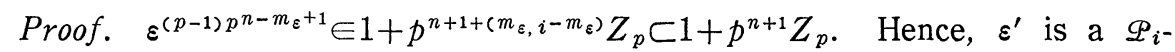
adic norm for $k_{n} / k$ at every $\mathscr{Q}_{\imath}$. By local class field theory, $\varepsilon^{\prime}$ is also a $\mathscr{P}$-adic norm at every prime $\mathscr{Q}$ of $k_{n}$ prime to $\not$. Then, by Hasse's norm theorem, $\varepsilon^{\prime}$ is a global norm for $k_{n} / k$. This implies $\varepsilon^{\prime} \in E \cap N_{n, 0}\left(k_{n}\right)=H$.

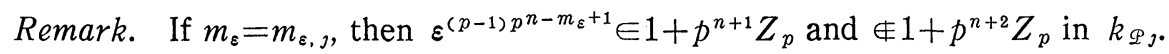

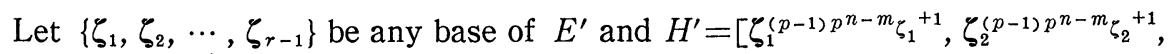

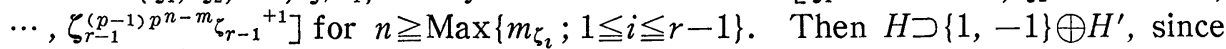

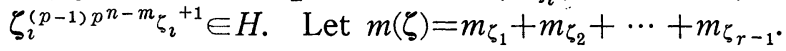

PROPOSITION 1. $m(\zeta) \leqq l_{n}+(r-1)$.

Proof. $[E: H]=p^{n(r-1)-l_{n}}$ and $\left[E:\{1,-1\} \oplus H^{\prime}\right]=(p-1)^{r-1} p^{n(r-1)-m(\zeta)+(r-1)}$. Here, $p^{n(r-1)-m(\zeta)+(r-1)} \geqq p^{n(r-1)-l_{n}}$, since $[E: H]$ divides $\left[E:\{1,-1\} \oplus H^{\prime}\right]$ and $(p-1)^{r-1}$ is prime to $p$. Then $m(\zeta) \leqq l_{n}+(r-1)$.

COROLLARY. $m(\zeta)$ remains bounded for all bases of $E^{\prime}$.

Proof. $m(\zeta) \leqq l_{n}+(r-1) \leqq l+(r-1)$.

Let $m=\operatorname{Max}\left\{m(\zeta)\right.$; any base $\left\{\zeta_{1}, \cdots, \zeta_{r-1}\right\}$ of $\left.E^{\prime}\right\}$ and, for $n \geqq m$, we take a 
base $\left\{\eta_{1}, \eta_{2}, \cdots, \eta_{r-1}\right\}$ of $E^{\prime}$ as in (1). Then $m(\eta) \leqq m$, but we can show that $m(\eta)=m$.

PROPOSITION 2. $\left|B_{n}\right|=p^{c+m-(r-1)}$ for $n \geqq m$.

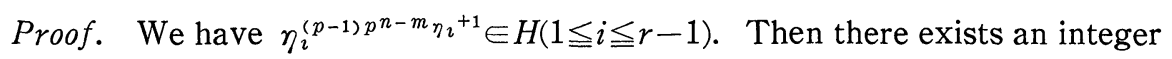
$d_{\imath}$ such that $\eta_{i}^{(p-1) p^{n-m} \eta_{\imath}+1}=\eta_{i}^{d_{i} p^{n-s_{i}}}$, so $d_{\imath}=(p-1) p^{d_{i}^{\prime}}$ for a non negative integer $d_{i}^{\prime}$. On the other hand, $\eta_{i}^{(p-1) \cdot p^{n-s i}} \in 1+p^{n+1} Z_{p}$ and $\eta_{i}^{(p-1) p^{n-m_{\eta_{i}}+1}}$ is a topological generator of $1+p^{n+1} Z_{p}$ in $k_{\mathscr{P}_{j}}$, where $m_{\eta_{i}}=m_{\eta_{i}, j^{*}}$. Hence, $d_{\imath}^{\prime}=0$, so $n-m_{\eta_{\imath}}+1$ $=n-s_{\imath}$. Then we have $[E: H]=p^{n(r-1)-m(\eta)+(r-1)}$ and $l_{n}=m(\eta)-(r-1)$. By proposition $1, m \leqq l_{n}+(r-1)=m(\eta)$. Hence, $m=m(\eta)$ and $\left|B_{n}\right|=p^{c+l_{n}}=p^{c+m-(r-1)}$.

This proposition was proved by Fukuda and Komatsu [1] in a real qudratic case. We can also prove the following result by the same way as in the theorem of Fukuda and Komatsu [1].

THEOREM. If $N_{m-1,0}\left(E_{m-1}\right)=E$ and the class number of $k$ is prime to $p$, then $\lambda_{p}(k)=0$, where $E_{n}$ is the unit group of $k_{n}$ and $\lambda_{p}(k)$ is the Iwasawa invariant of $k$ for the $p$.

Let $\left\{\zeta_{1}, \zeta_{2}, \cdots, \zeta_{r-1}\right\}$ be any base of $E^{\prime}$ and $n_{\zeta, \imath}=\operatorname{Max}\left\{m_{\zeta_{,}, \imath} ; 1 \leqq j \leqq r-1\right\}$ for $\mathscr{Q}_{\imath}$. Then $n_{\zeta, \imath}$ does not remain bounded for all bases of $E^{\prime}$.

Example. Let $k$ be a real cubic field and $\left\{\varepsilon_{1}, \varepsilon_{2}\right\}$ a base of $E^{\prime}$. Assume that

and

$$
\begin{aligned}
& \varepsilon_{1}^{p-1}=1+x p^{a_{1}}+\cdots \\
& \varepsilon_{2}^{p-1}=1+y p^{a_{2}}+\cdots
\end{aligned}
$$

in $k_{\mathscr{P}_{i}}$, where $1 \leqq x, y \leqq p-1$ and $a_{2} \geqq a_{1}$. We take a non negative integer $z$ such that

$$
z x \equiv-y(\bmod . p) \text { and } 1 \leqq z \leqq p-1 \text {. }
$$

Let $\varepsilon_{2}^{\prime}=\varepsilon_{1}^{z a_{2}-a_{1}} \varepsilon_{2}$. Then $\left\{\varepsilon_{1}, \varepsilon_{2}^{\prime}\right\}$ is a base of $E^{\prime}$ and $m_{\varepsilon_{2}^{\prime}, \imath}>a_{2}=m_{\varepsilon_{2}, \imath} \geqq a_{1}=m_{\varepsilon_{1}, \imath}$.

\section{REFERENCES}

[1] T. Fukuda And K. Komatsu, On the $\lambda$ invariants of $Z_{p}$-extensions of real quadratic fields, J. Number Theory, 23 (1986), 238-242.

[2] R. GREENBERG, On the IWASAWA invariants of totally real number fields, Amer. J. Math., 98 (1976), 263-284.

[3] H. YoKoI, On the class number of relatively cyclic number field, Nagoya Math. J., 29 (1967), 31-44.

Department of Mathematics
School of Science and Technology
Meiji University
Tama-Ku, KaWASaki
Kanagawa
214 Japan

